\title{
Road Accident Reconstruction Using On-board Data, Especially Focusing on the Applicability in Case of Autonomous Vehicles
}

\author{
Krisztian Pinter $^{1 *}$, Zsolt Szalay ${ }^{1}$, Gabor Vida ${ }^{1}$ \\ 1 Department of Automotive Technologies, Faculty of Transportation Engineering and Vehicle Engineering, Budapest University \\ of Technology and Economics, H-1521 Budapest, P. O. B. 91, Hungary \\ * Corresponding author, e-mail: krisztian.pinter@edu.bme.hu
}

Received: 19 November 2018, Accepted: 19 November 2018, Published online: 26 March 2020

\begin{abstract}
The application of Event Data Recorder (EDR) in passenger cars and vans has been compulsory in the USA since 2014. In the European Union, every passenger car and vehicle manufactured and released must have e-call systems since April 2018. However, neither the data recorded in Event Data Recorders regulated by the current standards nor the data recovered from e-call systems are enough to reconstruct the movements of the vehicle before and after the accident to a degree that the accident could be analyzed in the perspective of liability. The continuous expansion of autonomous vehicle functions - which will inevitably lead to completely autonomous vehicles - makes it particularly justifiable that all vehicles should possess EDR functions and that these data recorders shall store the satisfactory number of parameters for the vehicle's full movement reconstruction.

In the article, we will present a process of defining a data package - which will include a definition process for both the data points and the frequency of measuring and recording - that enables the post-event reconstruction of the full motion process, the vehicle movements and the evaluation of liability issues in both regular and irregular operation of autonomous and partially autonomous vehicles.
\end{abstract}

Keywords

accident reconstruction, Event Data Recorder (EDR), liability, autonomous vehicle

1 Introduction - the accident analysis, the emerging issues of accountability evaluation

By the appearance of the first road vehicles, the first traffic accidents started happening. Today, when the world's vehicle stock exceeds 1.2 billion, in 2015, there were about 1.25 million accidents with fatal consequences and an estimated amount of 30 million accidents that concluded with physical injuries to a person. In the European Union this year, there have been approximately 135000 accidents with fatal or serious injuries which costs (damage to property, treatment and rehabilitation) are estimated around a 100 billion Euros (WHO, 2015).

For the reasons mentioned above it is obvious that the reconstruction of the accident process is unavoidable for the clarification of liability questions. The analysis and exploration of the occurring accidents and their causation can be also of significant value in order to improve transport infrastructure and vehicle safety.

Traditionally, accident analysis is based on the data and traces generated during and collected after the irregular operation of the vehicle, which consist of:
- break and drift traces;

- collision traces (fallen debris, spilled fuel, scratch marks);

- traces created during the run-off phase after the collision (drift traces, scratch marks);

- end position of the vehicles;

- damage of the vehicles;

- people involved in the accident (vehicle operators, pedestrians);

- statements of witnesses.

The goal of accident analysis is the reconstruction of the whole accidental process, as in the whole motion process of all vehicles involved in the interval of a several seconds before and after the accident's trigger point (generally a collision). If the vehicles' motion process can be reconstructed with an acceptable precision, the motion series of the people involved (vehicle operators, pedestrians) which led to the accident can be examined, as well as 
the actions that could have been done in order to avoid it (i.e. when the situation became recognizable, perceptible) (Ghadi et al., 2018).

Considering all the above, the technical responsibility can be examined, which means that it can be determined whether the incorrect or late actions of the people involved or a reason beyond their control has led to the accident, for example a malfunction or shortage in the transport infrastructure or in one of the vehicles. It can be also examined if the people's action involved in the situation that potentially led to the accidents had any opportunities to avoid the accident as well as what actions they performed considering their objective opportunities (i.e. the delays are evaluable).

Considering all the above we can state that, some of the vehicle dynamic parameters recorded during the operation of the vehicle (the trace, speed, position of the vehicle) and certain maneuvers of controlling the vehicle (operation of the brake pedal, steering parameters, the state of lighting systems) used in the case of post-factum accident reconstruction are stored in the classic, currently available Event Data Recorders.

With the appearance of autonomous vehicle functions the question of responsibility can be examined in a wider spectrum than in the case of traditional (human-driven) vehicles, since the influence of these affected listed below needs to be clarified:

- driver,

- operator, manufacturer (OEM),

- system supplier (TIER1),

- operator of transport infrastructure.

The nature of change in general responsibility ratio is demonstrated in Fig. 1.

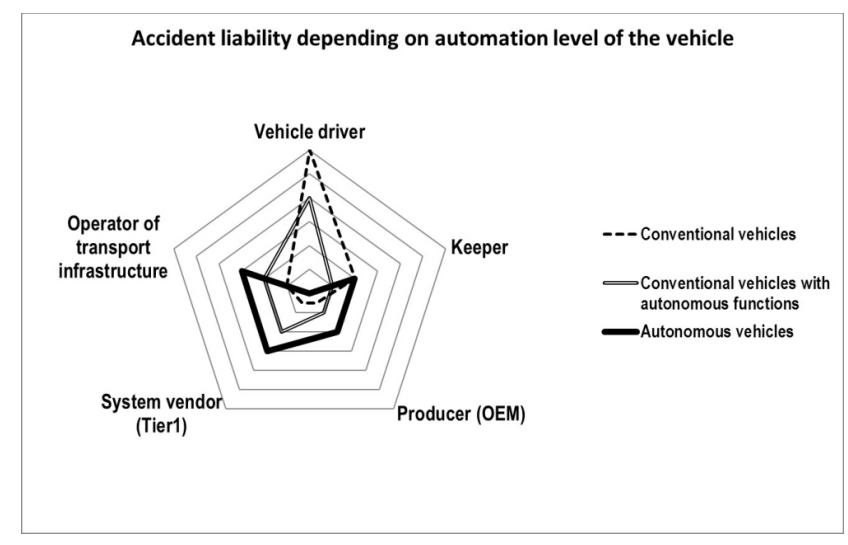

Fig. 1 The change of responsibility ratio in case of traditional and autonomous vehicles
Considering the significant expansion in the circle of potentially affected people in accidental liability we can state that the data storage of accidental even data recorders need to be expanded in order to examine certain questions during the accident analysis later such as who drove the vehicle at the time of the accident and beforehand when the take-over happened and what information the vehicle received from other transport participants (Pinter et al., 2017).

Our goal was to create an accidental data recorder that can support accident reconstruction and other analytical data in different levels of autonomity and can help with the examination of liability questions.

\section{Expedient data-set of EDR for autonomous vehicle}

In August 2006, the NHTSA had issued regulation 49 CFR Part 563, which did not include the obligation to install an EDR system in the vehicle at the time. However it included fifteen data-points that the EDR systems must record, stated in Table 1 (NHTSA, 2006).

We can observe that the minimal, obligatory data-set of the EDR includes for example the speed of the vehicle 5 seconds before the accident on a frequency of $2 \mathrm{~Hz}$ but does not include for example the longitudinal and lateral acceleration: this concludes that the motion process of the vehicle (course and other vehicle dynamic parameters) cannot be reconstructed.

Regulation 49 CFR Part 563 also determines an expanded data-set too: in case the manufacturer optionally - logs data form this type in the EDR system, it needs to measure and store these data-point according to the regulation. In this expanded data set, we can find the longitudinal and lateral accelerations, but the system only need to store it in the interval of $250 \mathrm{~ms}$ after the collision on the frequency of $100 \mathrm{~Hz}$ - this way these acceleration data-points are not acceptable for the reconstruction of the motion process prior to the event. However, the expanded data-set also includes parameters that can be used for the reconstruction of the whole motion process and the post-factum accident analysis such as information about the operation of the ABS or route-stabilizer, the steering angle or the tilt of the vehicle (rotation around axis $x$ ) - those latter three needs to be logged by the EDR system 50 seconds prior to the event.

During the determination of the data set in case of an EDR system suitable for the target goal, we need to consider the following boundary values: 
Table 1 Obligatory data-set of EDR systems (NHTSA, 2006)

\begin{tabular}{|c|c|c|}
\hline & Data element & Recording interval/timel (relative to time zero) \\
\hline 1 & Delta-V, longitudinal & 0 to $250 \mathrm{~ms}$, or 0 to End of Event Time plus $30 \mathrm{~ms}$ \\
\hline 2 & Maximum delta-V, longitudinal & 0 to $300 \mathrm{~ms}$, or 0 to End of Event Time plus $30 \mathrm{~ms}$ \\
\hline 3 & Time, maximum delta-V & 0 to $300 \mathrm{~ms}$, or 0 to End of Event Time plus $30 \mathrm{~ms}$ \\
\hline 4 & Speed, vehicle indicated & -5.0 to $0 \mathrm{sec}$ \\
\hline 5 & Engine throttle, $\%$ full (or accelerator pedal, $\%$ full) & -5.0 to $0 \mathrm{sec}$ \\
\hline 6 & Service brake, on/off & -5.0 to $0 \mathrm{sec}$ \\
\hline 7 & Ignition cycle, crash & $-1.0 \mathrm{sec}$ \\
\hline 8 & Ignition cycle, download & At time of download \\
\hline 9 & Safety belt status, driver & $-1.0 \mathrm{sec}$ \\
\hline 10 & Frontal air bag warning lamp, on/off & $-1.0 \mathrm{sec}$ \\
\hline 11 & $\begin{array}{l}\text { Frontal air bag deployment, time to deploy, in the case of a single stage air bag, or } \\
\text { time to first stage deployment, in the case of a multi-stage air bag, driver }\end{array}$ & Event \\
\hline 12 & $\begin{array}{l}\text { Frontal air bag deployment, time to deploy, in the case of a single stage air bag, or } \\
\text { time to first stage deployment, in the case of a multi-stage air bag, right front } \\
\text { passenger. }\end{array}$ & Event \\
\hline 13 & Multi-event, number of events $(1,2)$ & Event \\
\hline 14 & Time from event 1 to 2 & As needed \\
\hline 15 & Complete file recorded (yes, no) & Following other data \\
\hline
\end{tabular}

- Using the data recorded we need to able to reconstruct unequivocally the movements of the vehicle at least 180 seconds before, and 60 seconds after the accidents, meaning that we consider the vehicle as a rigid body in space and know all six of its degrees of freedom in every instant.

- Using data that is available in the vehicle.

- On a modern vehicle's on-board network, there are large sums of data that needs to be transported even today, but in the case of cooperative and autonomously operated vehicles, the amount of data traffic grows exponentially. Taken the finite capacity of each signal processing and storing unit into consideration we need to decrease the measuring and logging frequency of the EDR to a minimum value.

Considering the boundary values above we can examine in each case of data-sets, how the motion process can be reconstructed, and we need to create the processing algorithms and display the reconstructed vehicle motion process.

We evaluate the reconstructed path with attention to the degree of difference from the actual path and based on the analysis we can evaluate these data-sets and processing algorithms in regards of quality. The development process can be seen in Fig. 2.

Development of the model is happening simultaneously with the development of the vehicle, so the datasets that can be included in the EDR functions are clearly defined - since they are needed for the realization of an autonomous vehicle, they are obviously attainable in the vehicle (Bardos et al., 2017).

\section{Track reconstruction with on-board data - vehicle dynamics approach}

The data originated from this stage of the development for the sake of quicker implementation and for easier more clear reconstruction - we generate using vehicle dynamic simulation software. The simulation software we create a vehicle-motion process, and then with exporting the data taken into consideration we create the EDR data packages that later we will process. The processing and evaluating algorithms are developed in MATLAB environment and we also demonstrate the reconstructed motion process in MATLAB with 2D trajectories and using Simulink 3D Animation.

Since many of the vehicles at the present has on-board navigation systems and it is also indispensable for the operation of e-call system - and an autonomous vehicle cannot be realizable without a navigation system the GPS data can be used as basic input parameters for the reconstruction of the vehicle's motion process (Aldimirov and Arnaudov, 2018).

The geological coordinates data retrieved from the GPS unit in actual application are converted into rectangular coordinates using 3D spatial similarity transformation, the data retrieved form the coordinates $(x, y, z)$ created during the development process by vehicle simulation 


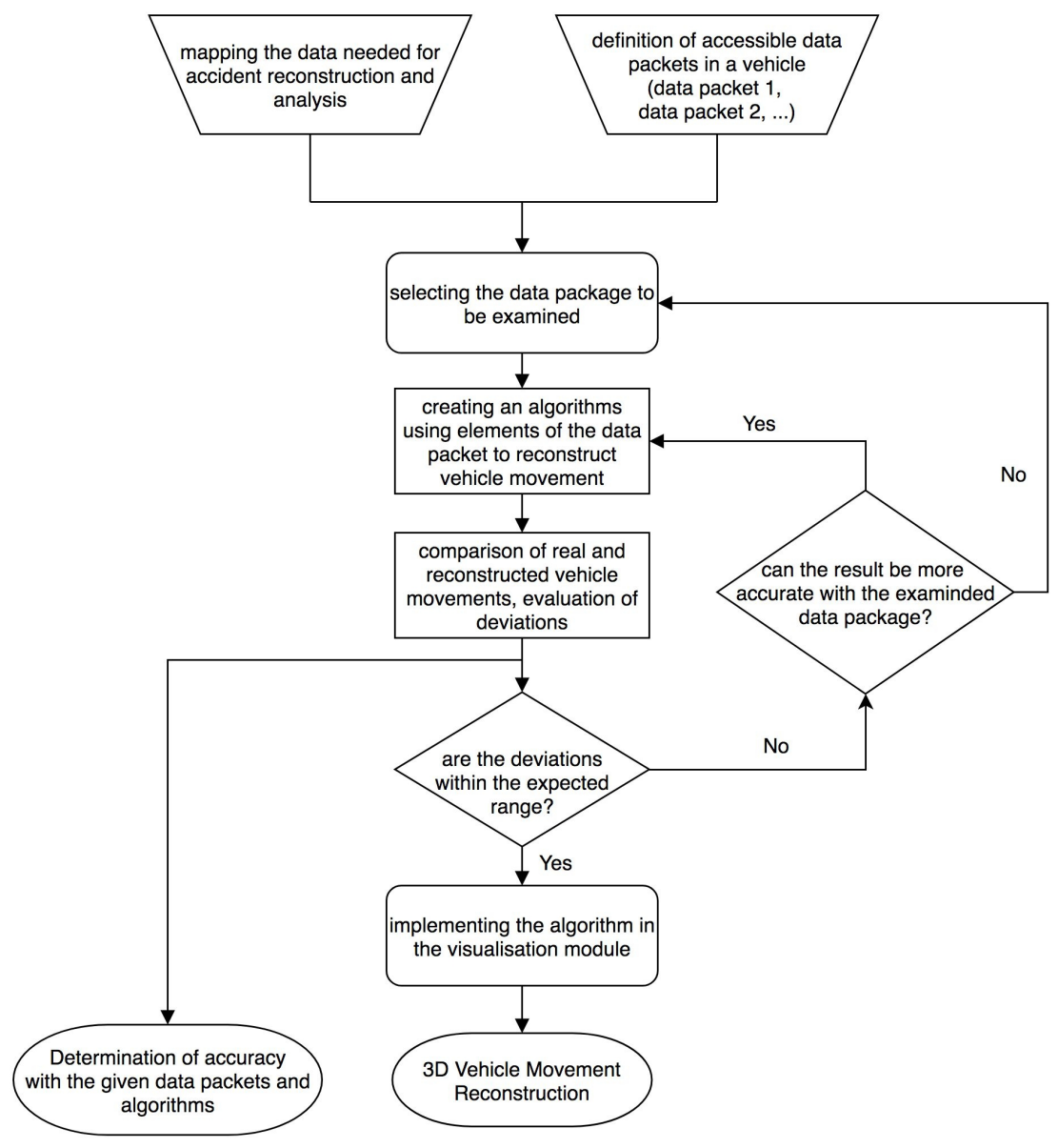

Fig. 2 The algorithm used for model development

software is exported and these coordinates are used as the data logged by the EDR.

Since the main goal of the development is to determine the minimal storing frequency, we use coordinate data logged at the frequency of $0.4 \mathrm{~Hz}$ - the vehicle's actual motion process with the logged positions are illustrated in Fig. 3.

Since in accordance to the earlier statements, the examination of vehicle movements - and if necessary, the accident analysis - is clearly relevant in a comprised time and consequentially space, for the display of vehicle movements, relative position coordinates are used - the coordinates recorded during the measurements are used for the vector $\boldsymbol{x}$ and $\boldsymbol{y}$ :

$x_{r e l, i}=x_{i}-\min (\overline{\boldsymbol{x}})$ and $y_{r e l, i}=y_{i}-\min (\overline{\boldsymbol{y}})$.

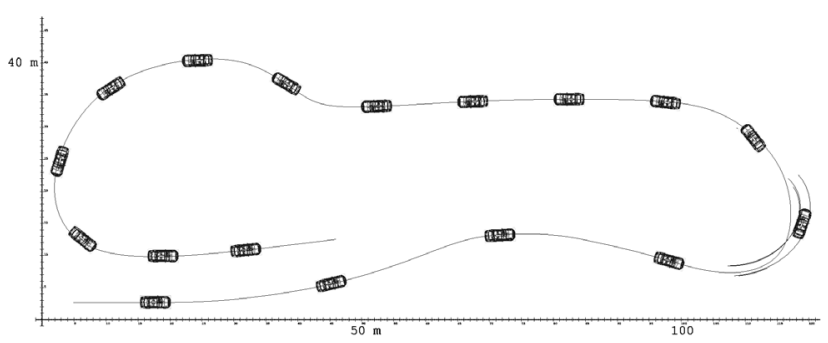

Fig. 3 The vehicle's actual motion process, logged at $0.4 \mathrm{~Hz}$
The first data package inspected only has the GPS coordinates of the vehicle's center of gravity, and in this case for the 3D display of the direction vector (heading) of the vehicle motion, we use the consecutive positions in each point:

$\Psi_{i}=\operatorname{arctg}\left(x_{i+1}-x_{i}\right) /\left(y_{i+1}-y_{i}\right)$.

If we move the vehicle along the line dictated by the heading between two measure points, it is clear that we are not demonstrating the actual movements of the vehicle, and it would display unnaturally sharp turns at certain points.

For the approximation of the missing data between two measured points we inspected two different methods. In the first method, we applied the vehicle dynamic approximation presented below. For the reconstruction of the vehicle's trajectory between the measured points we created as many intermediate points as we needed so the density corresponding to a desired sampling frequency in which the position of the vehicle is to be represented in our example, this frequency is $2 \mathrm{~Hz}$. If we used the mark $s p$ to represent the spacing of the generated points between two measured points, we can determine the coordinates of 
the intermediate points between the $j^{\text {th }}$ and the $(j+1)^{\text {th }}$ measured points with the following equation:

$$
\begin{gathered}
\left.x_{\text {gen }, j \rightarrow j+1}(i)\right|_{i=0} ^{s p-1}=x_{\text {norm }}(j)+\frac{\Delta x_{\text {norm } j, j+1}}{s p} * i \\
\left.y_{\text {gen }, j \rightarrow j+1}(i)\right|_{i=0} ^{s p-1}=y_{\text {norm }}(j)+\frac{\Delta y_{\text {norm } j, j+1}}{s p} * i .
\end{gathered}
$$

Next step is to determine the $x$ and $y$ components of the speed vector in each point:

$$
\begin{aligned}
& \left.v_{x, \text { gen }, j \rightarrow j+1}(i)\right|_{i=0} ^{s p-1}=\frac{x_{g e n, j \rightarrow j, j+1}(i+1)-x_{g e n, j \rightarrow j, j+1}(i+1)}{t_{g e n(i+1)}-t_{g e n}(i)} \\
& \left.v_{y, g e n, j \rightarrow j+1}(i)\right|_{i=0} ^{s p-1}=\frac{y_{g e n, j \rightarrow j, j+1}(i+1)-y_{g e n, j \rightarrow j, j+1}(i+1)}{t_{\operatorname{gen}(i+1)}-t_{g e n}(i)} .
\end{aligned}
$$

This way, the speed vector in each point of the generated vehicle movement is known, and it can be determined that at certain points, how much average acceleration is required for the vehicle. Since we used linear interpolation for the generated intermediate points between the measured points, it is clear that the value of the acceleration vector between two generated points is 0 , however between the last generated point of the given section and the measured end-point - if the vehicle is not using straight-line smooth motion - we can determine the actual acceleration of the vehicle.

The speed and acceleration components retrieved between the $4^{\text {th }}, 5^{\text {th }}$ and $6^{\text {th }}$ point of the examined motion process by the method described above is presented in Fig. 4.

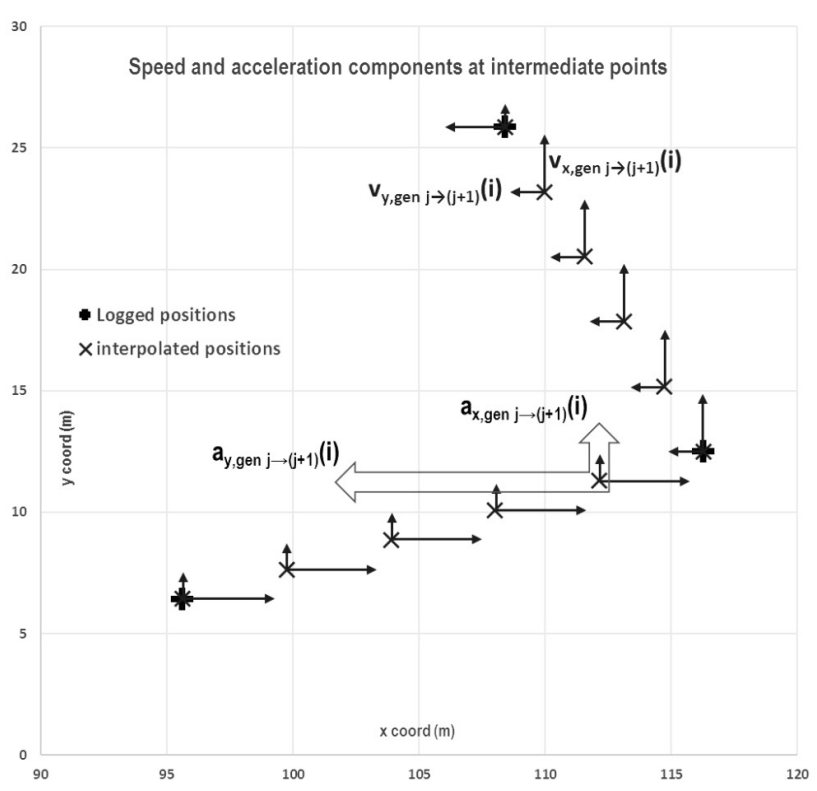

Fig. 4 The speed and acceleration components between measured points
In reality, the vehicle does not move straight between the two measured points but at a certain arc, so according to this there should be acceleration vectors with a value of non-zero between two generated points between the measured points.

Consequentially, in the section between two measured points, the acceleration vector with the salient value would not occur between the last generated point and the endpoint of the section but at a given section or a certain point of that section - that is determined by the physically possible acceleration - is where the speed change would happen. Considering that with this measurement method we only know the position of the vehicle at discrete points and the time elapsed between point, with the lack of information we determine the acceleration between intermediate points so the time integral between the last intermediate point and the end point of the section added by using the logic above is the same as the integral between all the other intermediate points of the section, while in each point the acceleration components are changing at an even rate. This way we basically divide the speed change occurring in the last sections in the whole section between the two measured points as a sort of approximation of real change in speed between the measured points, illustrated in Fig. 5.

By integrating the acceleration data generated in the intermediate points according to the method above, the speed and the positions can be calculated, and the trajectory of the vehicle is probable.

The calculated trajectory in the intermediate points compared to the recorded trace line is illustrated in Fig. 6.
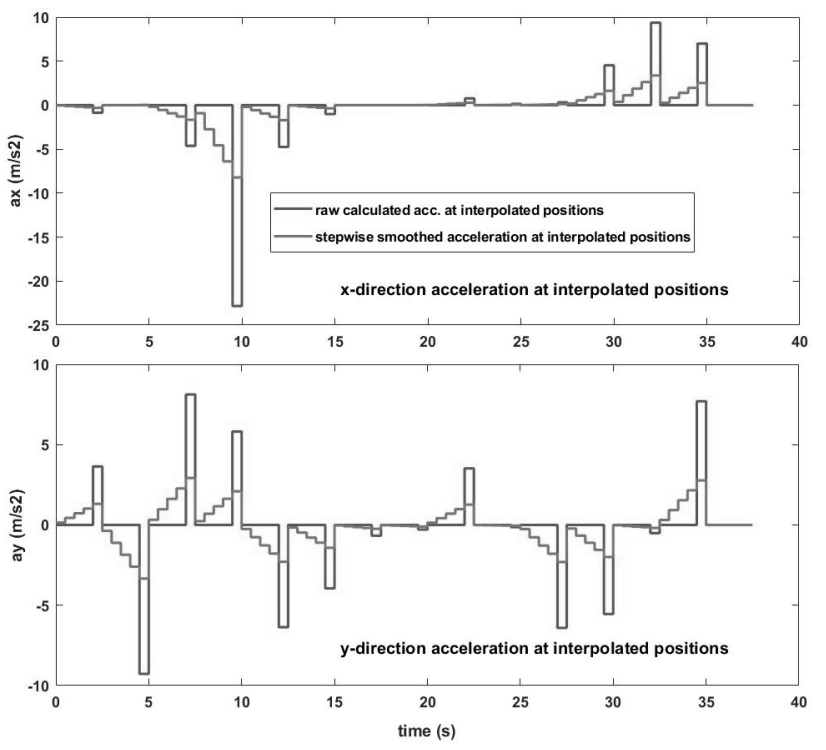

Fig. 5 Crude, stepped-smooth acceleration components in intermediate points 


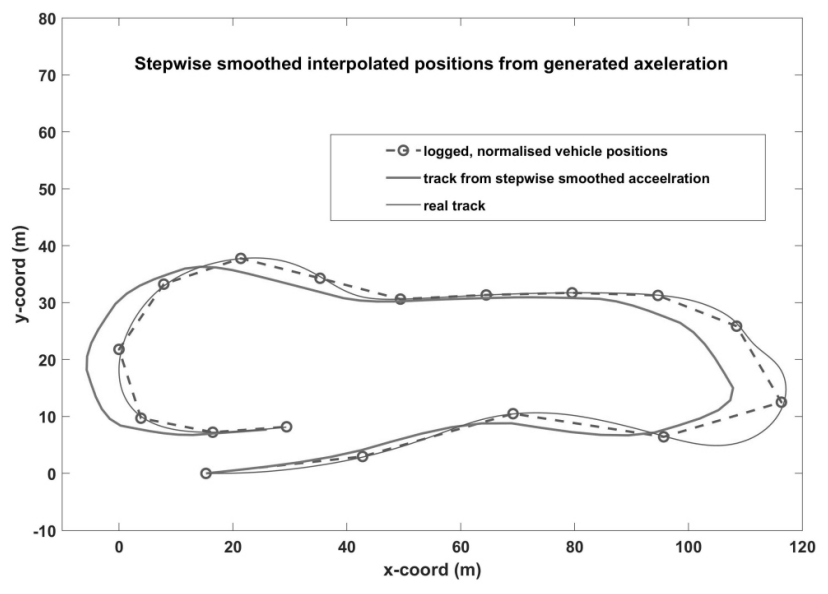

Fig. 6 Trajectory calculated from the stepped-smooth acceleration data

In Fig. 6 we can see that the method is more inaccurate as arc in the vehicle's trajectory is less in the examples we observed (7-11) and significant difference between the real and the calculated speed values occur between second $(28,35)$, at $(80-110)$ and $(220,250)$ displacements, when the vehicle moves by the two edges of the trajectory in Fig. 6.

According to the tests concluded we can state the presented method - when we integrate the vehicle's acceleration from the data points recorded and stored on low frequency - at the trajectory inspected, the result is approximately $-6.4 \mathrm{~m}$ difference between the reconstructed and the real position change on every $100 \mathrm{~m}$ while the maximum difference between the calculated and real positions is $11.03 \mathrm{~m}$ and $4.13 \mathrm{~m}$ in directions $x$ and $y$.

In the case of the real trace line on sections with more traceable, but smaller arcs, when turns are narrower - arcs with smaller radius - the real trajectory is not suitably traceable by the generated trajectory.

\section{Track reconstruction with on-board data - a geometric approach}

In the next part, we will try to reconstruct the real trace of the vehicle by align curves on the measured and stored positions. Since we also often use third-degree splines in vehicle trajectory planning, we can investigate the accuracy of a third-degree interpolation in approximating a trace line depending on the measurement and storage frequencies.

The started data is the same trajectory as before, positions recorded at a $0.4 \mathrm{~Hz}$ frequency. The positions recorded with the aligned curves by third-degree interpolation and the real trajectory is illustrated in Fig. 7.

Concluded the tests we performed, it can be stated that the with data recorded and stored at the frequency of $0.4 \mathrm{~Hz}$, we reconstruct the trajectory with third-degree

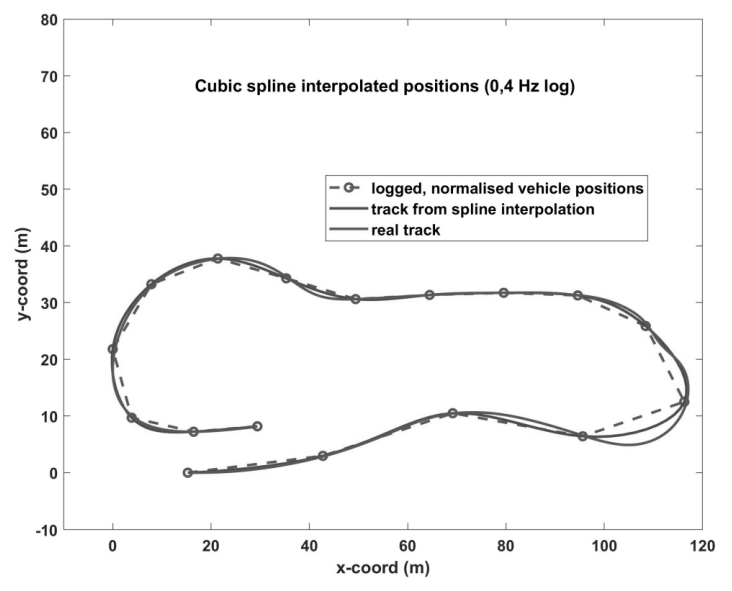

Fig. 7 The trajectory by spline interpolation $(0.4 \mathrm{~Hz} \log )$

spline interpolation, the result is a difference of $0.9 \mathrm{~m}$ difference in a 100 meters on average, while the maximum difference between the calculated and real positions is $5.2 \mathrm{~m}$ and $2.2 \mathrm{~m}$ in directions $x$ and $y$.

Next, we will investigate that with the spline interpolation at which frequency and resolution applied, we can get the accuracy required in our application.

In the next test concluded, we recorded the position of the vehicle at the frequency of $1 \mathrm{~Hz}$ - the positions recorded with the aligned curves by third-degree interpolation are illustrated in Fig. 8.

We can conclude that in the case of the data recorded at a frequency of $1 \mathrm{~Hz}$, the difference of the measured track and the position change calculated from the spline interpolation is $0.12 \mathrm{~m}$ while the maximum difference is $1.91 \mathrm{~m}$ and $0.14 \mathrm{~m}$ between the measured and calculated positions. Average difference on the whole trajectory is $0.04 \mathrm{~m}$ and $0.001 \mathrm{~m}$ in positions $x$ and $y$.

By these results, we can conclude that in regular operation, the trajectory of the vehicle can be reconstructed with a

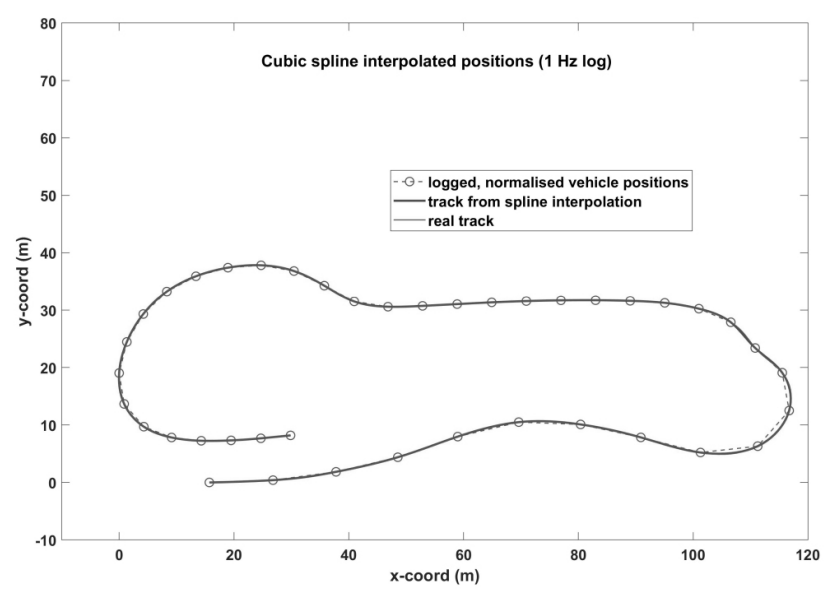

Fig. 8 The trajectory determined with spline interpolation (1 Hz log) 
satisfactory accuracy by third-degree spline interpolation while using data recorded at a $1 \mathrm{~Hz}$ frequency. The larger difference between the real and the reconstructed trajectory occurs and the start and at the end of the recording, though this can be managed by redefining the log intervals. The required recording frequency in irregular operation will need further tests to be determined.

\section{Conclusion}

The compulsory data content of the accident data recorders set out in the current standard and the data obtained from e-call systems are not sufficient to reconstruct the movement of the vehicle before and after the accident at a level that can be used to analyze the accident process and to investigate liability issues. In the case of an autonomous vehicle function, responsibility issues - occurring in connection with an accident, - more widely investigated than in conventional (man-driven) vehicles. Taking into account the significant increase in the number of entities potentially affected by accidents, it can be stated that the extension of data content of accident data recorders is necessary. To investigate liability issues, reconstruction

\section{References}

Aldimirov, M., Arnaudov, R. (2018) "Method for automated reconstruction of a car's path during crash from GPS/INS data using a Kalman filter", Advances in Engineering Software, 115, pp. 386-390. https://doi.org/10.1016/j.advengsoft.2017.10.009

Bardos, A., Vass, S., Nyerges, Á., Tihanyi, V., Szalay, Zs. (2017) "Path tracking controller for automated driving", In: Vehovszky, B., Takács, J., Bán, K. (eds.) 34 th $^{\text {th }}$ Inernational Colloquium on Advanced Manufacturing and Repairing Technologies in Vehicle Industry, Visegrad, Hungary, pp. 9-12.

Ghadi, M., Török Á., Tánczos, K. (2018) "Integration of Probability and Clustering Based Approaches in the Field of Black Spot Identification", Periodica Polytechnica Civil Engineering. https://doi.org/10.3311/PPci.11753 and analysis of the accident is indispensable, and in connection this, it is necessary to examine that using essential on-board data, how successful can be reconstructed the regular or irregular motion of the vehicle. Based on the studies presented in this article it can be stated that based on the logged GPS coordinates of the vehicle's center of gravity, vehicle movements during regular operation can be reconstructed with sufficient accuracy even at $1 \mathrm{~Hz}$ measurement frequency, using geometric methods. At the same time, the vehicle movement during the irregular operation cannot be determined only from the positions of the center of gravity and together with the extension the range of data needed for proper reconstruction it is also appropriate to increase the measurement and storage frequency. Results of our research are used to determine the data content of accident data recorders suitable for use in vehicles at different levels of autonomous driving.

\section{Acknowledgement}

The project has been supported by the European Union, co-financed by the European Social Fund. EFOP-3.6.2-16-2017-00002.

National Highway Traffic Safety Administration (NHTSA) (2006) "49 CFR Part 563 Event Data Recorders", NHTSA, Washington, USA.

Pinter, K., Szalay, Zs., Vida, G. (2017) "Liability in Autonomous Vehicle Accidents", Communications - Scientific Letters of the University of Zilina, 19(4), pp. 30-35.

SAE (2014) "SAE J3016 Taxonomy and Definitions for Terms Related to On-Road Motor Vehicle Automated Driving Systems", SAE International, Warrendale, PA, USA. https://doi.org/10.4271/J3016_201401

World Health Organization (WHO) (2015) "Global Status Report on Road Safety", WHO, Geneva, Switzerland. 